\title{
DNALK2 inhibits the proliferation and invasiveness of breast cancer MDA-MB-231 cells through the Smad-dependent pathway
}

\author{
$\mathrm{KE} \mathrm{WANG}^{1,2}$, XIAOXIAO SUN ${ }^{2}, \mathrm{HONG} \mathrm{LEI} \mathrm{FENG}{ }^{2}, \mathrm{CHANG} \mathrm{FEI}^{2}$ and YAN ZHANG ${ }^{2}$ \\ ${ }^{1}$ Yongchuan Hospital, Chongqing Medical University, Chongqing 402160; ${ }^{2}$ Key Laboratory of Diagnostic Medicine \\ Designated by the Chinese Ministry of Education, Chongqing Medical University, Chongqing 400016, P.R. China
}

Received June 24, 2016; Accepted August 9, 2016

DOI: $10.3892 / o r .2016 .5343$

\begin{abstract}
Breast cancer is one of the most common malignant neoplasms diagnosed in females worldwide. Bone morphogenetic proteins (BMPs), which belong to the TGF- $\beta$ superfamily, regulate a wide range of cellular responses including cell proliferation, differentiation, adhesion, migration and apoptosis in breast cancer. BMPs can bind to type I and II serine/threonine kinase receptors to regulate cell proliferation, invasion, migration, and apoptosis. Type I receptors are expressed in various breast cancer cell lines and primary tumor samples. Activin-like kinase 2 (ALK2) is generally expressed in breast cancer cells (MDA-MB-231, MCF7, SK-BR-3 and MDA-MB-468); however, the effect of ALK2 on the proliferation and metastasis of breast cancer cells remains unknown. We used a dominant-negative mutant of ALK2 to research the function of ALK2. We aimed to ascertain whether dominant-negative mutant ALK2 adenovirus vector (DNALK2) receptors can compete with wild-type ALK2 receptors. The present study showed that DNALK2 inhibited the growth, migration and metastasis of breast cancer cells by inhibiting the SMAD-dependent pathway and downregulating connective tissue growth factor and inhibitor of differentiation 1 expression, in vivo and in vitro. These observations indicate that ALK2 is a potential therapeutic agent for the treatment of breast cancer.
\end{abstract}

\section{Introduction}

Breast cancer is one of the most common malignant neoplasms diagnosed in women worldwide. Metastasis, the single greatest cause of morbidity and mortality in breast cancer patients, commonly occurs in the bones, lungs and liver (1-4). However, the molecular mechanisms underlying breast cancer cell proliferation, migration and metastasis still require further

Correspondence to: Professor Yan Zhang, Key Laboratory of Diagnostic Medicine Designated by the Chinese Ministry of Education, Chongqing Medical University, 1 Yixue Yuan Road, Yuzhong, Chongqing 400016, P.R. China

E-mail: zy2753@hotmail.com

Key words: DNALK2, breast cancer, growth, CTGF, ID1 investigation, and there is still no effective treatment strategy for breast cancer metastasis.

Bone morphogenetic proteins (BMPs) regulate a wide range of cellular responses including cell proliferation, adhesion, migration and apoptosis $(5,6)$. BMPs bind to heterodimers of type I and II serine/threonine kinase receptors. Upon ligand binding, the constitutively active type II receptor transphosphorylates and activates the kinase activity of the type I receptor. Once active, the type I receptor phosphorylates the intracellular effector proteins, SMAD1, SMAD5, and SMAD8, which have complex involvement with a common partner, SMAD4 to accumulate in the nucleus and regulate the expression of genes involved in cell growth, cell differentiation, cell apoptosis, cellular homeostasis and other cellular functions $(7,8)$. This pathway is known as the SMADdependent pathway. BMP ligands bind to different receptors in different contexts. The physiological association with a specific receptor depends on both the binding affinity and the actual availability of the ligand and the receptor in a specific environment. Type I receptors determine the specificity of the intracellular response (9). Activin-like kinase 2 (ALK2; ACVR1), ALK3 (BMPR1A), and ALK6 (BMPR1B) are type I receptors known to mediate BMP signaling.

Research has shown that type I receptors (ALK3 and ALK6) are expressed in various breast cancer cell lines and primary tumor samples. Expression of dominant-negative ALK3 in a mouse xenograft model was found to result in decreased invasiveness and metastasis (10). ALK6 is associated with the poor prognosis of breast cancer patients. In ER-positive breast cancer specimens, ALK6 expression was found to correlate with high tumor grade and high tumor proliferation. Reduced expression of ALK6 was found to correlate with poor prognosis and increased proliferation of breast cancer cells (11).

ALK2 belongs to the type I receptors, but the function of ALK2 in breast cancer is unknown. Research has reported that ALK2 is generally expressed in various types of cells (12). ALK2 gene mutation was found to lead to ossificans progressiva fibrodysplasia (FOP) in bone disease (13), and led to Down's syndrome. The reason for congenital heart defects in Down's syndrome patients is due to ALK2 gene mutation which alters the BMP/SMAD pathway (14). In mesenchymal stem cells, ALK2 is necessary for the signaling pathway of BMP9-induced osteogenic (15). It has also been reported that ALK2 promotes the proliferation of lens cells in the early stage of mouse embryonic 
development, but inhibits proliferation in late stage (16). ALK2 exhibits various functions in different types of cells and even in the same type of cells, yet the reason is unknown (17). Recently, research has focused on the relationship between ALK2 and tumors. BMP9 binds to ALK2 to promote ovarian cancer by activating the SMAD1/4 signaling pathway $(18,19)$. ALK2 mutant was found to promote the growth of prostate cancer PC3-M cells (20). However, the effect of ALK2 on the proliferation and metastasis of breast cancer cells is still unknown.

Dominant-negative mutation uses engineering technology to mutate the specific receptor and overexpress the dominant-negative receptor to produce a negative regulatory effect. Our group cloned the ALK2 extracellular domain cDNA to the adenovirus vector, and successfully constructed the dominant-negative mutant ALK2 adenovirus vector (DNALK2) . DNALK2 receptors can compete with wild-type ALK2 receptors by binding to ligands, and inhibit ligands to active cell signaling pathways.

\section{Materials and methods}

Cell culture and infection. The human breast cancer cell lines MDA-MB-231 and MDA-MB-468 (Shanghai Institute for Biological Sciences, Chinese Academy of Sciences, Shanghai, China) were maintained in L-15 medium supplemented with $10 \%$ fetal bovine serum (FBS; Gibco, Grand Island, NY, USA) at $37^{\circ} \mathrm{C}$ without $\mathrm{CO}_{2}$. Other breast cancer cell lines, MCF-7 and SK-BR-3 (Shanghai Institute for Biological Sciences, Chinese Academy of Sciences) were cultured in Dulbecco's modified Eagle's medium (DMEM) supplemented with $10 \%$ FBS at $37^{\circ} \mathrm{C}$ with $5 \% \mathrm{CO}_{2}$.

Log-phase MDA-MB-231 cells were seeded at a density of $2 \times 10^{6} /$ bottle. The cells were infected with RFP-expressing and recombinant DNALK2 adenovirus vehicles $24 \mathrm{~h}$ later. After 8-12 h of cultivation, the medium was replaced with fresh medium. The fluorescence was then observed $24 \mathrm{~h}$ later. The recombinant MDA-MB-231/DNALK2 cells were used for subsequent experiments. The experimental cells were divided into the MDA-MB-231 and MDA-MB-231/RFP control groups as well as the MDA-MB-231/DNALK2 group.

RNA isolation and semi-quantitative RT-PCR. Total RNA was extracted from artificially induced breast cancer cell samples using TRIzol (Invitrogen Life Technologies, Carlsbad, CA, USA).

The first-strand cDNA was synthesized from $1 \mu \mathrm{g}$ of total RNA using the M-MLV kit (Promega Corp., Madison, WI, USA). ALK2 gene expression was quantified by semi-quantitative reverse transcription-polymerase chain reaction (RT-PCR). $G A P D H$ was used as an endogenous control. Gene expression analysis was performed with Quantity One software (Bio-Rad, Berkeley, CA, USA). The PCR conditions were $94^{\circ} \mathrm{C}$ for $5 \mathrm{~min}$, followed by 30 cycles at $94^{\circ} \mathrm{C}$ for $30 \mathrm{sec}, 52^{\circ} \mathrm{C}$ for $20 \mathrm{sec}$, and $72^{\circ} \mathrm{C}$ for $10 \mathrm{sec}$. The mRNA expression levels of the target gene were normalized to those of $G A P D H$.

The specific primers for $A L K 2$ were 5'-GGCTGCTTCCA GGTTTAT-3' (forward) and 5'-CATACTGCGAACACTAC AGA-3' (reverse). Those for GAPDH were 5'-CAGCGACACC CACTCCTC-3' (forward) and 5'-TGAGGTCCACCACCC TGT-3' (reverse).
Western blot analysis. The protein concentrations in the cell lysates were determined using a kit (RIPA, P0013B; Bi Yuntian Biological Technology Institution, Shanghai, China). Equal amounts of proteins were separated by sodium dodecyl sulphate-polyacrylamide gel electrophoresis and blotted onto nitrocellulose membranes. The proteins were then probed with rabbit polyclonal anti-SMAD1/5/8 (sc-6031; Santa Cruz Biotechnology, Inc., Santa Cruz, CA, USA), rabbit polyclonal anti-phospho-SMAD1/5/8 (AB3848; Millipore Corp., Billerica, MA, USA), rabbit monoclonal anti-inhibitor of differentiation 1 (ID1) (ab134163), and rabbit polyclonal anti-connective tissue growth factor (CTGF) antibodies (ab6992) (both from Abcam) and peroxidase-conjugated secondary antibodies. A GAPDH protein sample was used as the control for equal protein loading. Protein bands were visualized using Quantity One software.

Quantitative real-time $R T$-PCR. Quantitative real-time RT-PCR was performed as previously described (21). All samples were run in duplicate for each experiment. The following primer sequences were used for CTGF (sense, 5'-GCGGCTTACCGACTGGA-3' and antisense, 5'-AGG CGGCTCTGCTTCTC-3'); ID1 (sense, 5'-CGGTCTCAT TTCTTCTCG-3' and antisense, 5'-TCGGTCTTGTTCTCC CTC-3'); and GAPDH (sense, 5'-CAGCGACACCCACTC CTC-3' and antisense, 5'-TGAGGTCCACCACCCTGT-3'). The relative expression of target mRNAs was normalized to the reference gene GAPDH using the $2^{-\Delta \Delta C T}$ method and is expressed as the fold-change relative to the control $(0.1 \%$ or DharmaFECT1) (21).

Cell viability assay. The MTT assay was performed in quintuplicate to assess the viability of the recombinant MDA-MB-231/DNALK2 cells. Approximately $1-3 \times 10^{4}$ of cells from each group in $500 \mu \mathrm{l}$ of medium were seeded in 24-well plates. Approximately $50 \mu 1$ of MTT reagent $(5 \mathrm{mg}$ / $\mathrm{ml}$; Promega Corp.) was added, and the mixture was incubated for $4 \mathrm{~h}$. After addition of $450 \mu \mathrm{l}$ of dimethyl sulfoxide, absorbance was measured daily for the following 5 days at $492 \mathrm{~nm}$ using a microplate reader. Then, a growth curve was drawn.

Colony forming assay. Log-phase cells were collected and seeded in triplicate in a soft agar medium at 200 cells/culture dish for 10-14 days. When the clones were able to be observed, the cells were washed twice by PBS and stained by the Wright method. The colony-forming rate was calculate as: (Colony number/seeded cell number) x 100\%. Each experiment was repeated thrice.

Wound closure assay. Log-phase cells were collected and seeded at $5 \times 10^{5} /$ well in 6 -well plates. On the following day, the cells were infected with recombinant DNALK2 adenovirus for $24 \mathrm{~h}$, and a wound was created at the center of the culture using a pipette tip. Photographs were taken under a microscope immediately and $36 \mathrm{~h}$ after the incision was made. The incision width of the different sites was measured, and the average wound closure rate was calculated. The wound-closure rate was calculated as: [ 0 - $\mathrm{h}$ incision width - 36-h incision width)/0-h incision width] x $100 \%$. 

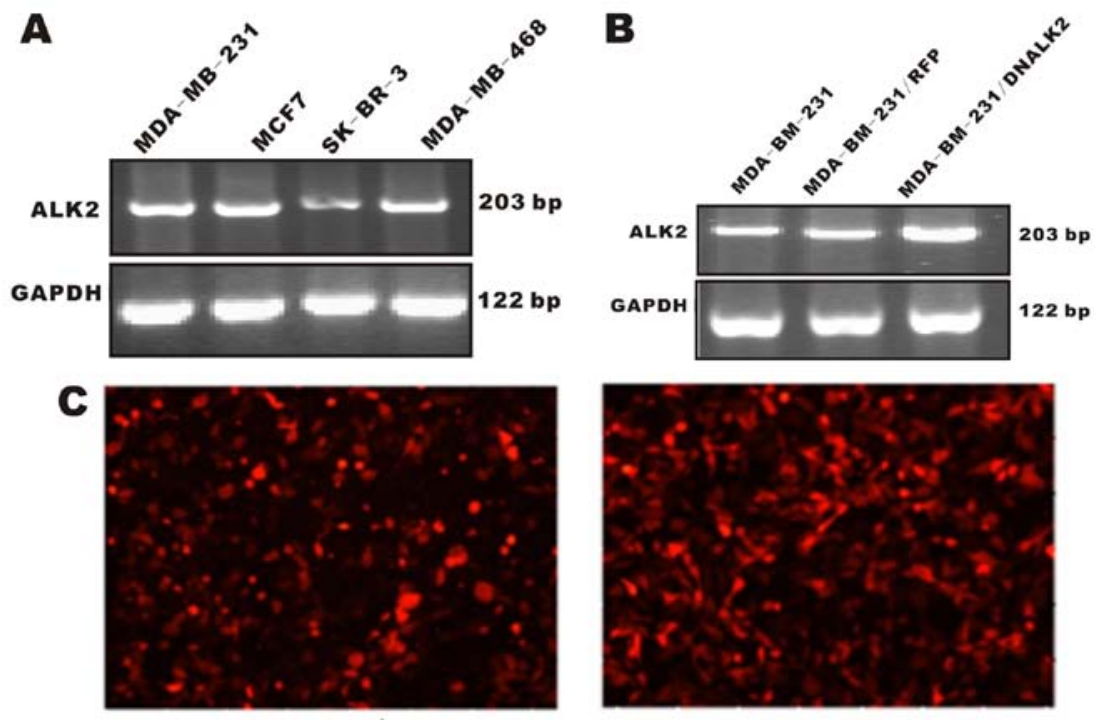

MDA-MB-231/RFP

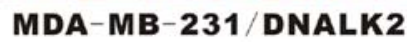

Figure 1. Recombinant MDA-MB-231/DNALK2, dominant-negative ALK2, was established (A) ALK2 mRNA expression in different kinds of breast cancer cells was detected by RT-PCR. (B) Dominant-negative ALK2 expression in MDA-MB-231/DNALK2 cells was significantly increased after infection, and DNALK2 transcripts were increased in the MDA-MB-231/DNALK2 cells, compared with the MDA-MB-231/RFP and MDA-MB-231 cells. (C) Infection efficiency of MDA-MB-231 cells infected with dominant-negative ALK2 adenovirus for $24 \mathrm{~h}$ observed under a fluorescence microscope (x100).

Transwell invasion assay. The invasion assay was performed as previously described (15). The cells were seeded at $2 \times 10^{5} /$ well in duplicate in the upper chamber of type I collagen-coated 24-well culture inserts. After $24 \mathrm{~h}$, the cells were dried for 5 min, fixed with dehydrated alcohol, and stained with hematoxylin and eosin. The cells that invaded the collagen-coated inserts were counted. Mean values for five randomly selected fields were obtained for each well. The experiments were performed 4-fold and repeated thrice. Values are expressed as means \pm standard errors.

Animals. Four-week-old female nude mice (Balb/c, Beijing, China) were used. All experiments were approved by the Institutional Animal Care and Use Committee of Chongqing Medical University as well as regional authorities [reference no. SYXK(YU)2007-0001] in accordance with the 'Guidelines for the Welfare of Animals in Experimental Neoplasia' (the China Coordinating Committee on Cancer Research). The animals were housed in individual ventilated cages under sterile conditions, and were given free access to food and water. At the end of the experimental period, the animals were sacrificed by cervical dislocation.

Animal models. Female nude mice were randomly divided into three groups of five mice each. Two groups were implanted subcutaneously with $2 \times 10^{6}$ control MDA-MB-231 and MDA-MB-231/RFP cells, respectively. The third group was implanted subcutaneously with 2x106 MDA-MB-231/DNALK2 cells. After 13 days, when tumors were observable, the tumor diameters were recorded every three days. Tumor volume $\left(\mathrm{V}\right.$, in $\left.\mathrm{cm}^{3}\right)$ was then calculated as: $(4 \pi / 3) \times[(\mathrm{a}+\mathrm{b}) / 4]^{3}$, where $\pi=3.14$. The mice were sacrificed after three weeks, and the tumors were collected. The tumors were then dissected and processed for further histomorphometrical and immunohistochemical analyses.
Immunohistochemistry. Paraffin-embedded nude mouse xenograft breast tumors were sliced into $4-\mu \mathrm{m}$ sections and deparaffinized. The sections were rehydrated and heat-treated for antigen-retrieval with citric acid buffer as previously described. The sections were then incubated with rabbit antiSMAD1/5/8, rabbit anti-phospho-SMAD1/5/8, rabbit anti-ID1 and rabbit anti-CTGF antibodies and rabbit anti-PCNA for $1 \mathrm{~h}$ following standard protocol. Staining procedures were performed under standardized conditions. The sections were counterstained with Gill's hematoxylin, mounted, and coverslipped. Staining intensity was independently assessed by the authors. SMADD1/5/8, phospho-SMAD1/5/8, ID1 and CTGF expression were determined with integrated optical density (IOD) using Image-Pro Plus 5.1. The nuclear staining revealed PCNA-positive cells, and PCNA expression was determined as: (PCNA-positive cells/total number of tumor cells) x $100 \%$.

Statistical analysis. Results are expressed as means \pm standard deviations. Relative CTGF and ID1 expression data were analyzed using real-time quantitative PCR and the $2^{-\Delta \Delta C T}$ method. All statistical analyses were performed by SPSS 19.0 using the independent sample t-test for comparing two sample groups. For all tests, $\mathrm{P}<0.05$ was considered statistically significant.

\section{Results}

ALK2 mRNA is detected in breast cancer cells. In the present study, four breast cancer cell lines were used to investigate ALK2 expression. Our group wanted to determine whether ALK2 expression in the human breast cancer cell lines is associated with tumorigenic potentials. ALK2 mRNA was detected in the four breast cancer cell lines by RT-PCR amplification (Fig. 1A). The result suggests that ALK2 expression is correlated with the progression and metastasis of breast cancer. 

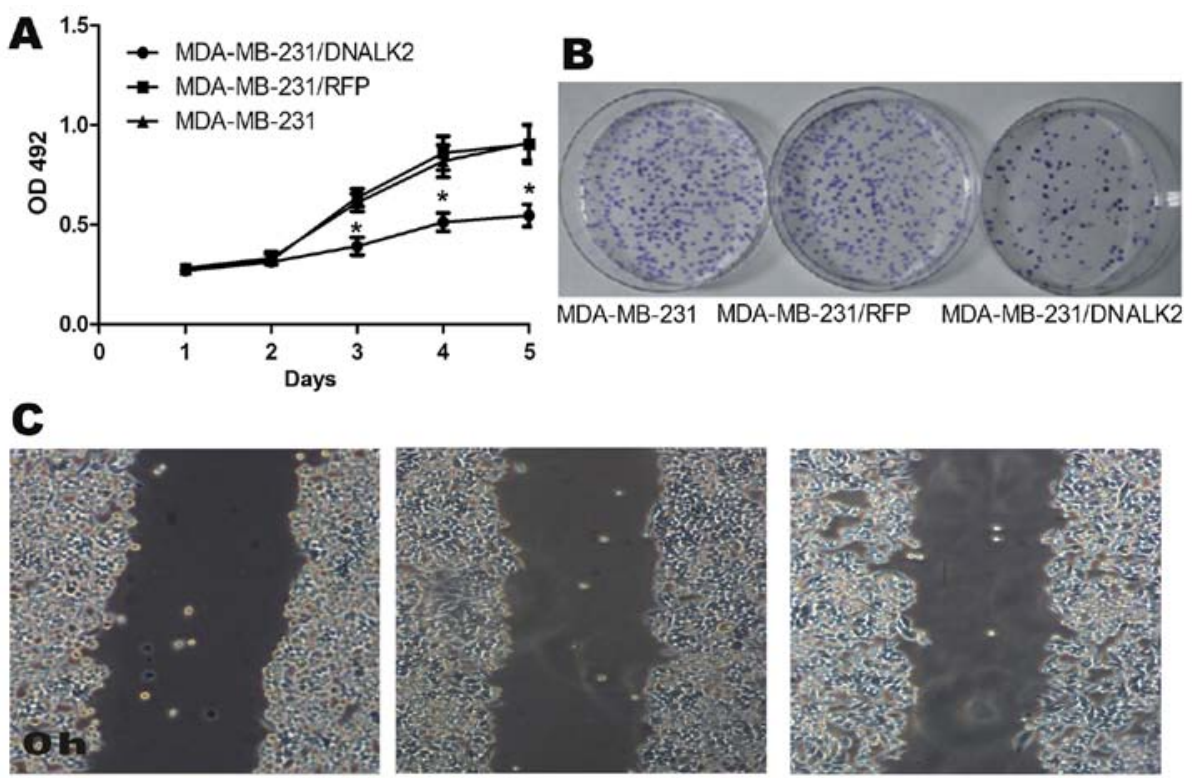

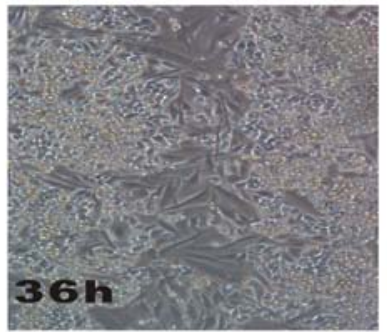

MDA-MB-231

D

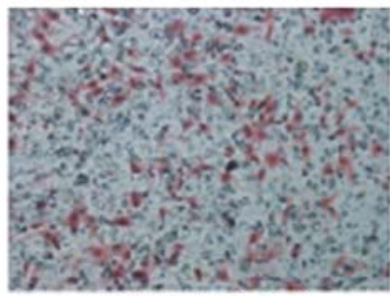

MDA-MB-231

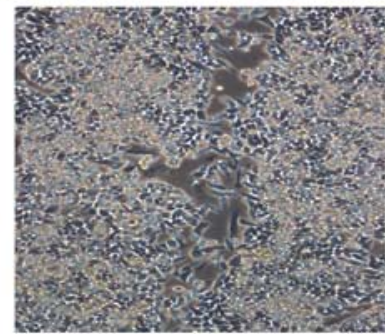

MDA-MB-231/RFP

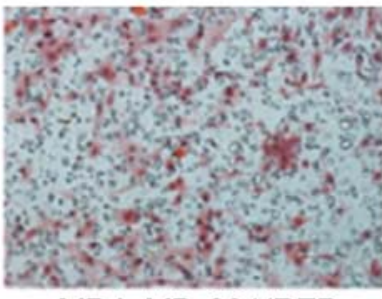

MDA-MB-231/RFP

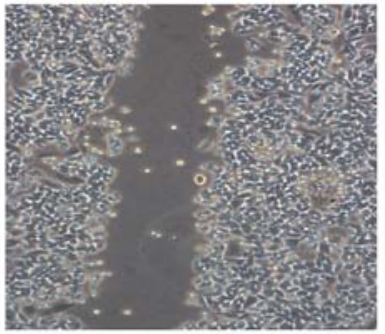

MDA-MB-231/DNALK2

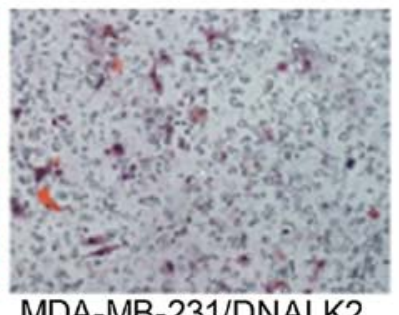

Figure 2. Decreased growth, migration, and invasiveness of MDA-MB-231/DNALK2 cells caused by dominant-negative ALK2. (A) The effects of dominant-negative ALK2 on the proliferation of MDA-MB-231 cells. Dominant-negative ALK2 decreased the proliferation of MDA-MB-231 cells. ${ }^{*} \mathrm{P}<0.05$. (B) The colony-forming assay results from the various groups. The colony-forming rate decreased significantly in the MDA-MB-231/DNALK2 cells. (C) The wound closure assay results from the various groups. The wound closure rate was apparently decreased in the MDA-MB-231/DNALK2 cells (x100). (D) The Transwell invasion assay results from the various groups. The number of invasive cells was notably decreased in the MDA-MB-231/DNALK2 cells (x100).

Recombinant MDA-MB-231/DNALK2 cells are established. MDA-MB-231 cells were used in the present study to generate a dominant-negative ALK2 recombinant cell line to examine the roles of ALK2 in the proliferation and invasiveness of breast cancer cells. The dominant-negative ALK2 recombinant MDA-MB-231/DNALK2 cells were established by infection with the DNALK2 adenovirus. MDA-MB-231/RFP cells infected with an empty adenovirus vector were also constructed as control cells. The RT-PCR results showed that these recombinant cells were well established for use in the subsequent experiments (Fig. 1B and C).

DNALK2 inhibits the proliferation and invasiveness of breast cancer cells in vitro. ALK2 was reported to bind to BMP9 to promote the proliferation of ovarian cancer cells but ALK2 was found to inhibit the growth and to induce the apoptosis of prostate cancer cells. In the present study, we elucidated the effects of ALK2 on breast cancer cells in vitro by analyzing cell proliferation, migration, and invasion abilities. MTT and colony-forming assays were used to detect the cell proliferation ability. The results showed that the proliferation of MDA-MB231/DNALK2 cells was decreased from $0.8951 \pm 0.0902$ (MDA-MB-231/RFP) and 0.9012 \pm 1.0021 (MDA-MB-231) to $0.5563 \pm 0.0617$ (MDA-MB-231/DNALK2) (Fig. 2A). Accordingly, Fig. 2B shows that the colony-forming rate of MDA-MB-231/DNALK2 cells was decreased from $91.3 \pm 3.6 \%$ (MDA-MB-231/RFP) and 93.9 $\pm 5.6 \%$ (MDA-MB-231) to $34.0 \pm 5.3 \%$ (MDA-MB-231/DNALK2). These results indicated that DNALK2 inhibited the growth of MDA-MB-231 breast cancer cells.

Wound closure and Transwell invasion assays were used to detect the invasion and migration abilities of the 

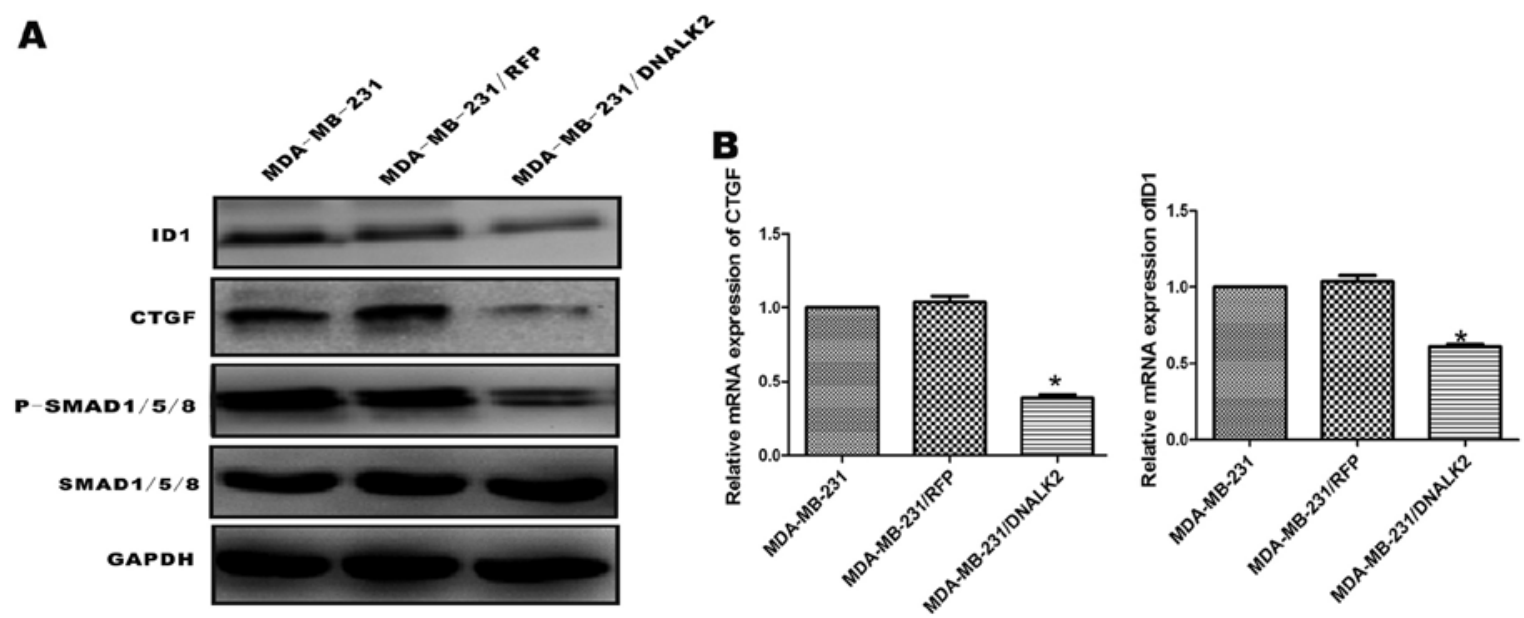

Figure 3. DNALK2 inhibits the BMP/SMAD signaling pathway and downregulates CTGF and ID1 expression in MDA-MB-231/DNALK2 cells. (A) MDA-MB231 cells were incubated for $48 \mathrm{~h}$ with the dominant-negative ALK2 adenovirus and western blot analysis was performed with the indicated antibodies. Western blot analysis with anti-SMAD1/5/8, p-SMAD1/5/8, CTGF and ID1 antibodies showed that p-SMAD1/5/8, CTGF and ID1 expression in the MDA-MB-231 cells was downregulated after culturing for $48 \mathrm{~h}$ with dominant-negative ALK2. GAPDH expression was used as the internal control. (B) Transcript levels of CTGF and CXCR4 in the MDA-MB-231/DNALK2 cells were detected using real-time RT-PCR analysis. GAPDH was used as the internal control. ${ }^{*}<<0.05$.

MDA-MB-231/DNALK2 cells. Fig. 2C shows that the wound closure rate decreased from $94.4 \pm 3.6 \%$ (MDA-MB-231) and $91.7 \pm 2.9 \%$ (MDA-MB-231/RFP) to $41.2 \pm 5.7 \%$ (MDA-MB231/DNALK2) $(\mathrm{P}<0.05)$. Fig. 2D shows that invasive cell numbers decreased from 142.7 \pm 16.1 (MDA-MB-231) and 135.3 \pm 15.8 (MDA-MB-231/RFP) to $35.5 \pm 6.7$ (MDA-MB231/DNALK2) $(\mathrm{P}<0.05)$.

DNALK2 inhibits the BMP/SMAD signaling pathway and downregulates IDI and CTGF expression in MDA-MB-23I cells. In a previous study, DNALK2 was found to inhibit the growth, migration, and invasiveness of MDA-MB-231 breast cancer cells. To establish whether the BMP/SMAD signaling pathway is functional, the MDA-MB-231 cells were stimulated with DNALK2, and phosphorylation of SMAD1/5/8 was assessed using specific anti-phosphosite antibodies via immunoblot analysis. The recombinant MDA-MB-231/DNALK2 cells exhibited lower levels of phosphorylated SMAD1/5/8 than levels noted in the MDA-MB-231/RFP and MDA-MB-231 cells, while the total SMAD1/5/8 protein was similar in the three groups (Fig. 3A). ID1 and CTGF expression levels in the recombinant MDA-MB-231/DNALK2 cells were assessed using western blot analysis and real-time RT-PCR, which are reportedly related with the proliferation and invasion of tumor cells. CTGF protein $(40 \mathrm{kDa})$ and ID1 protein $(16 \mathrm{kDa})$ expression levels were also significantly lower in the MDA-MB-231/DNALK2 cells than these levels in the MDA-MB-231/RFP and MDA-MB-231 cells (Fig. 3A). ID1 and CTGF mRNA was downregulated in the MDA-MB-231/DNALK2 cells (Fig. 3B) $\left(\mathrm{CTGF}, 2^{-\Delta \Delta \mathrm{CT}}=0.39, \mathrm{P}<0.05 ; \mathrm{ID} 1,2^{-\Delta \Delta \mathrm{CT}}=0.63, \mathrm{P}<0.05\right)$.

DNALK2 inhibits tumor formation through the SMAD-dependent pathway in vivo. To investigate the effects of DNALK2 on the growth of breast cancer cells in vivo, three groups of cells (MDA-MB-231/DNALK2 as well as the two control cells MDA-MB-231/RFP and MDA-MB-231) were subcutaneously implanted into nude mice. After 13 days, tumors were observed in the mice implanted with the two control cell lines, but not in the mice implanted with the MDA-MB-231/DNALK2 cells. After 16 days, tumors were observed in the mice implanted with the MDA-MB-231/DNALK2 cells (Fig. 4A). Fig. 4B and C shows that the tumor volume was decreased from $2.005 \pm 0.351$ (MDA-MB-231) and 2.054 \pm 0.273 (MDA-MB-231/RFP) to $0.804 \pm 0.135$ (MDA-MB-231/DNALK2 group) on day 31 $(\mathrm{P}<0.001)$. Hence, dominant-negative ALK2 decreased the proliferation of breast cancer cells in nude mice and significantly decreased tumor burden over time. These results were confirmed by immunohistochemical analysis of PCNA expression in the tumor sections. The PCNA-positive cell rate decreased from 55.9 \pm 6.5 (MDA-MB-231) and 53.7 \pm 4.8 (MDA-MB-231/RFP) to $21.4 \pm 4.9 \%$ (MDA-MB-231/DNALK2 group) $(\mathrm{P}<0.001)$ as shown in Fig. 4D.

To establish whether the BMP/SMAD signaling pathway was functional in the MDA-MB-231/DNALK2 cells, total SMAD1/5/8, p-SMAD1/5/8, CTGF and ID1 was assessed using specific antibodies via immunohistochemistry analysis. The p-SMAD1/5/8, CTGF and ID1 protein expression was decreased in the MDA-MB-231/DNALK2 group compared with the MDA-MB-231/RFP and MDA-MB-231 groups as determine by immunochemical staining (Fig. 5). These data suggest that DNALK2 is a potent inhibitor of the growth and metastasis of breast cancer cells.

\section{Discussion}

ALK2 belongs to the TGF- $\beta$ superfamily type I receptors, which is expressed in various carcinoma cell lines of ovarian, prostate, pancreatic, and breast origin $(5,9,14)$. More recently, ALK2 has been linked to carcinogenesis and tumor progression. ALK2 has been described as a pleiotropic receptor in carcinoma cell regulation, dependent on the cell type, developmental stage, and microenvironment of the carcinoma cells. For example ALK2 acts as a tumor suppressor in prostate cancer and myeloma cells, but as an oncogene in ovarian and lung cancer cell lines $(8,18,19,22)$. BMP7 binds 
A

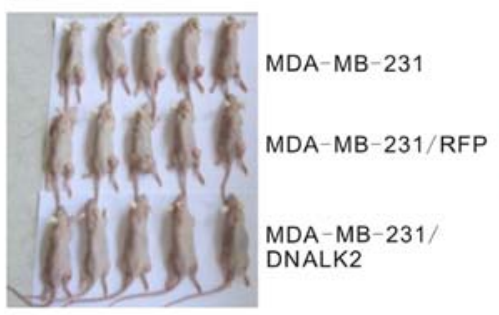

B

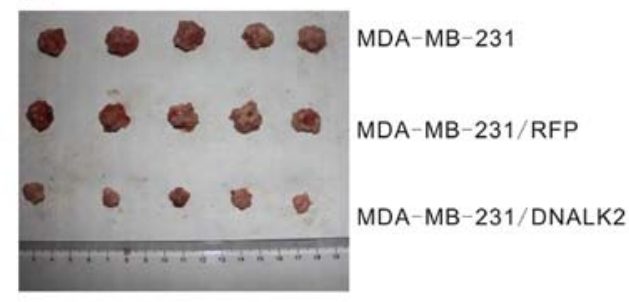

C

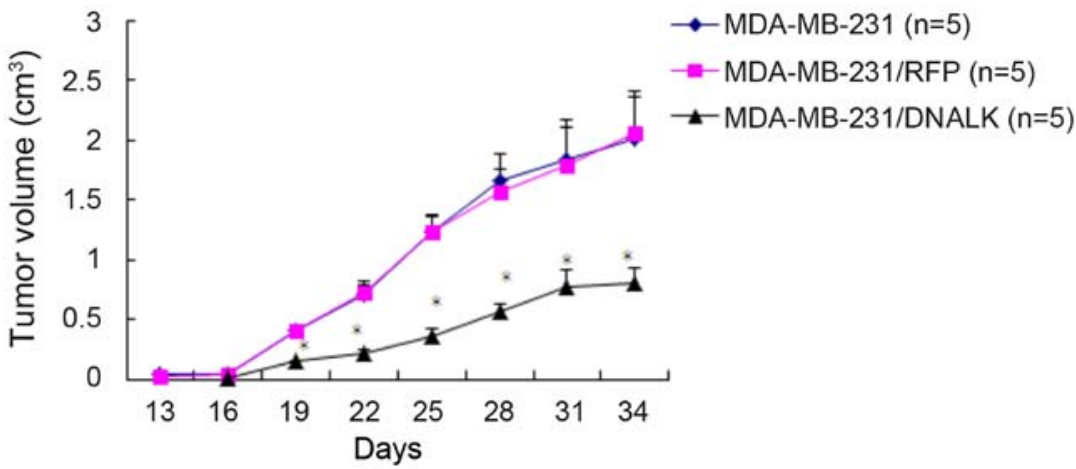

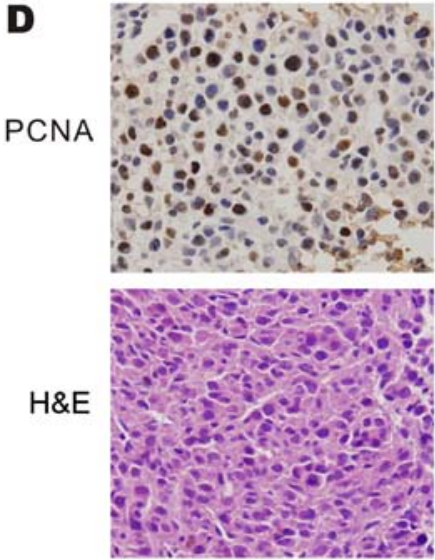

MDA-MB-231

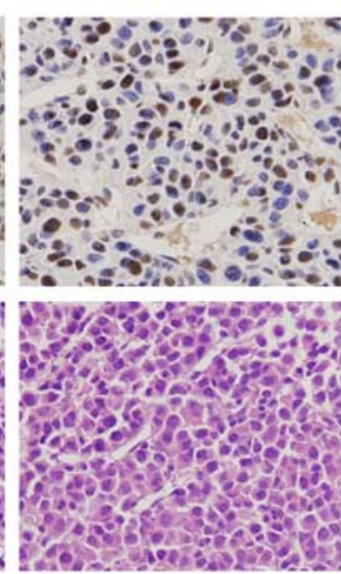

MDA-MB-231/RFP MDA-MB-231/DNALK2

Figure 4. Inhibition of tumor formation in vivo caused by dominant-negative ALK2. (A) Nude mice were sacrificed after 8 weeks, and the tumors were collected. (B) Tumor sizes in the various groups. (C) The tumor growth curves of the various groups. Tumor size was also quantified every 3 days by measuring hindlimb diameter. Tumor size of the MDA-MB-231/DNALK2 froup was significantly smaller at day 34 . ${ }^{*} \mathrm{P}<0.05$. (D) PCNA staining of tumor tissues of the various groups using immunochemical staining. The PCNA-positive cell rate was decreased in the MDA-MB-231/DNALK2 cells. The proliferation of MDA-MB-231 cells was inhibited by DNALK2 (x100).

to ALK2 to induce mesenchymal-epithelial transition (MET) in melanoma tumor cells, and ultimately inhibits metastasis by regulating downstream of Twist protein expression (23). BMP9 binds to ALK2 to activate the BMP/SMAD signaling pathway to enhance the proliferation of ovarian cancer cells (18).

Type I receptors (ALK3 and ALK6) are expressed in primary breast cancer samples even in several breast cancer cell lines. Dominant-negative ALK3 was found to result in decreased invasiveness and metastasis in a mouse xenograft model (16). Reduced expression of ALK6 increased proliferation of breast cancer cells (11). ALK2 belongs to the type I receptors, but the function of ALK2 in breast cancer is unknown. There are few studies concerning its function in the development of breast cancer.

In the present study, MDA-MB-231 cells were infected with AdDNALK2. MTT results showed that the number of
MDA-MB-231 cells began to decline on the third day after DNALK2 adenovirus infection, obviously decreased on the fifth day; we used a colony forming assay to determine that the cell colony number in the MDA-MB-231/DNALK2 group was significantly decreased compared to the control group, which further confirmed the MTT results. Wound closure assay showed that the wound healing rate of the MDA-MB-231/ DNALK2 cell group was significantly decreased compared to the MDA-MB-231/RFP cells. Transwell invasion assay showed that the number of invasive MDA-MB-231 cells pretreated with AdDNALK2 were obviously reduced compared with the control group, in vitro. These results showed that the cell proliferation, invasion and migration abilities of MDA-MB-231 cells were reduced after infection with the DNALK2 adenovirus. ALK2 is an important factor influencing breast cancer growth and metastasis and acts as a potential target for inhibiting the metastasis of breast cancer. 


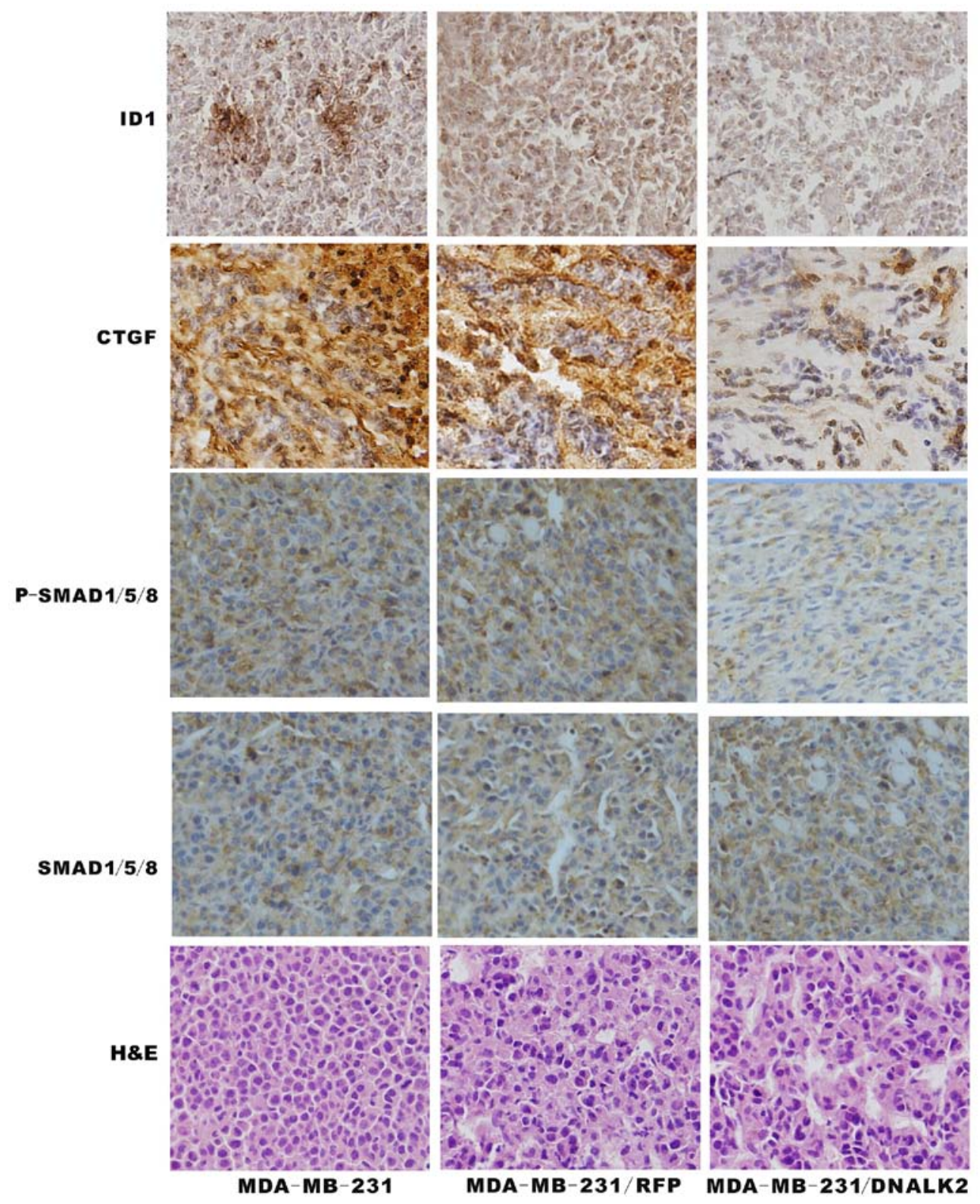

Figure 5. DNALK2 inhibits tumor formation through the SMAD-dependent pathway in vivo. Representative images of immunohistochemical staining of SMAD1/5/8, p-SMAD1/5/8, CTGF and ID1 in nude mouse breast cancer tissue samples (x100). p-SMAD1/5/8, CTGF and ID1 expression was downregulated after dominant-negative ALK2 in vivo.

CTGF, a new induced fibrosis factor, is a CCN (CTGF, Cyr61 and Nov) family member, and is associated with tumor proliferation and metastasis. It is involved in the occurrence of many diseases and tumors such as system scleroderma, atherosclerosis, liver fibrosis and biliary atresia (24-26), but the distribution and biological function of CTGF are different in different types of tumor tissues. It can regulate tumor progression positively or negatively. ALK2 acts as an oncogene in liver cell carcinoma, gastric cancer, glioma, breast cancer, esophageal adenocarcinoma resulting in reduced survival and poor patient prognosis (27), while in pancreatic cancer, esophageal squamous cell carcinoma, colorectal cancer, laryngeal squamous cell carcinoma, thyroid cancer and lung cancer, ALK2 acts as a tumor suppressor to inhibit tumor growth (28). CTGF and ID1 exhibit high expression in breast cancer, and their expression is correlated with tumor size, lymph node status and age; expression change in cancer cells and tissues is of great significance $(28,29)$. We found that CTGF and ID1 expression at the mRNA and protein levels was significantly reduced after DNALK2 infection in the MDA-MB-231 cells, suggesting that DNALK2 is likely to inhibit tumor proliferation, invasion and migration by downregulation of CTGF and ID1.

Animal models are important tools with which to research the biological behavior of tumor cells in vivo. Thus, we successfully constructed a breast cancer subcutaneous tumor model in nude mice and found that DNALK2 significantly inhibited the growth of breast cancer cells in nude mice. The expression of CTGF and ID1 was also downregulated in the MDA-MB231/DNALK2 group at the same time. The specific signaling pathway and related mechanism warrant further study. In the present study, one of the critical mechanisms underlying the inhibiiton of the growth and metastasis of breast cancer cells by DNALK2 is by reducing CTGF and ID1 expression to inhibit the BMP/SMAD cell signaling pathway. 
In conclusion, ALK2 is an important factor that can inhibit breast cancer growth, invasion and migration through classical BMP/SMAD cell signaling (BMPs, BMPRs Smads - gene transcription in the nucleus - corresponding protein production) (30), ALK2 has profound potential in the prognosis and therapy of breast cancer metastasis. Further investigation may lead to a novel therapeutic approach for breast cancer. Yet, we need further understanding of the processes involved in breast cancer metastasis and we must develop effective preventive strategies.

\section{Acknowledgements}

The present study was supported by the National Natural Science Foundation of China (grant no. 81172017), the Natural Science Foundation Project of Chongqing Dducation Committee (grant no. KJ1500202) and the Natural Science Foundation Project of Yong Chuan Hospital (grant no. YJQN201401/YJZQN201517).

\section{References}

1. Raida M, Clement JH, Leek RD, Ameri K, Bicknell R, Niederwieser D and Harris AL: Bone morphogenetic protein 2 (BMP-2) and induction of tumor angiogenesis. J Cancer Res Clin Oncol 131: 741-750, 2005

2. Lian WJ, Liu G, Liu YJ, Zhao ZW, Yi T and Zhou HY: Downregulation of BMP6 enhances cell proliferation and chemoresistance via activation of the ERK signaling pathway in breast cancer. Oncol Rep 30: 193-200, 2013.

3. Buijs JT, Petersen M, van der Horst G and van der Pluijm G: Bone morphogenetic proteins and its receptors; therapeutic targets in cancer progression and bone metastasis? Curr Pharm Des 16 1291-1300, 2010

4. Boeuf S, Bovée JV, Lehner B, van den Akker B, van Ruler M, Cleton-Jansen AM and Richter W: BMP and TGFbeta pathways in human central chondrosarcoma: Enhanced endoglin and Smad 1 signaling in high grade tumors. BMC Cancer 12: 488, 2012.

5. Ye L, Mason MD and Jiang WG: Bone morphogenetic protein and bone metastasis, implication and therapeutic potential. Front Biosci (Landmark Ed) 16: 865-897, 2011.

6. Guo D, Huang J and Gong J: Bone morphogenetic protein 4 (BMP4) is required for migration and invasion of breast cancer. Mol Cell Biochem 363: 179-190, 2012

7. Lim J, Tu X, Choi K, Akiyama H, Mishina Y and Long F: BMP-Smad4 signaling is required for precartilaginous mesenchymal condensation independent of Sox9 in the mouse. Dev Biol 400: 132-138, 2015.

8. Olsen OE, Wader KF, Misund K, Våtsveen TK, Rø TB, Mylin AK, Turesson I, Størdal BF, Moen SH, Standal T, et al: Bone morphogenetic protein-9 suppresses growth of myeloma cells by signaling through ALK2 but is inhibited by endoglin. Blood Cancer J 4: e196, 2014.

9. Lei R, Zhang K, Liu K, Shao X, Ding Z, Wang F, Hong Y, Zhu M, $\mathrm{Li} \mathrm{H}$ and $\mathrm{Li} \mathrm{H}$ : Transferrin receptor facilitates TGF- $\beta$ and BMP signaling activation to control craniofacial morphogenesis. Cell Death Dis 7: e2282, 2016

10. Brederlau A, Faigle R, Elmi M, Zarebski A, Sjöberg S, Fujii M, Miyazono K and Funa K: The bone morphogenetic protein type $\mathrm{Ib}$ receptor is a major mediator of glial differentiation and cell survival in adult hippocampal progenitor cell culture. Mol Biol Cell 15: 3863-3875, 2004

11. Bokobza SM, Ye L, Kynaston HE, Mansel RE and Jiang WG: Reduced expression of BMPR-IB correlates with poor prognosis and increased proliferation of breast cancer cells. Cancer Genomics Proteomics 6: 101-108, 2009.

12. Alsamarah A, LaCuran AE, Oelschlaeger P, Hao J and Luo Y: Uncovering molecular bases underlying bone morphogenetic protein receptor inhibitor selectivity. PLoS One 10: e0132221, 2015.
13. Herrera-Esparza R, Pacheco-Tovar D, Bollain-Y-Goytia JJ, Torres Del Muro F, Ramírez-Sandoval R, Pacheco-Tovar MG, Castañeda-Ureña $M$ and Avalos-Díaz E: An activin receptor IA/activin-like kinase-2 (R206H) mutation in fibrodysplasia ossificans progressiva. Case Rep Genet 2013: 260371, 2013.

14. Joziasse IC, Smith KA, Chocron S, van Dinther M, Guryev V, van de Smagt JJ, Cuppen E, Ten Dijke P, Mulder BJ, Maslen CL, et al: ALK2 mutation in a patient with Down's syndrome and a congenital heart defect. Eur J Hum Genet 19: 389-393, 2011.

15. Song T, Wang W, Xu J, Zhao D, Dong Q, Li L, Yang X, Duan X, Liang Y, Xiao Y, et al: Fibroblast growth factor 2 inhibits bone morphogenetic protein 9-induced osteogenic differentiation of mesenchymal stem cells by repressing Smads signaling and subsequently reducing Smads dependent up-regulation of ALK1 and ALK2. Int J Biochem Cell Biol 45: 1639-1646, 2013.

16. Chakkalakal SA, Zhang D, Culbert AL, Convente MR, Caron RJ, Wright AC, Maidment AD, Kaplan FS and Shore EM: An Acvrl R206H knock-in mouse has fibrodysplasia ossificans progressiva. J Bone Miner Res 27: 1746-1756, 2012.

17. Thomas PS, Sridurongrit S, Ruiz-Lozano P and Kaartinen V: Deficient signaling via Alk2 (Acvr1) leads to bicuspid aortic valve development. PLoS One 7: e35539, 2012.

18. Herrera B, van Dinther $M$, Ten Dijke P and Inman GJ: Autocrine bone morphogenetic protein-9 signals through activin receptor-like kinase-2/Smad1/Smad4 to promote ovarian cancer cell proliferation. Cancer Res 69: 9254-9262, 2009.

19. Tsai CL, Tsai CN, Lin CY, Chen HW, Lee YS, Chao A, Wang TH, Wang HS and Lai CH: Secreted stress-induced phosphoprotein 1 activates the ALK2-SMAD signaling pathways and promotes cell proliferation of ovarian cancer cells. Cell Rep 2: 283-293, 2012.

20. Romero D, Terzic A, Conley BA, Craft CS, Jovanovic B, Bergan RC and Vary CP: Endoglin phosphorylation by ALK2 contributes to the regulation of prostate cancer cell migration. Carcinogenesis 31: 359-366, 2010.

21. Wang K, Feng H, Ren W, Sun X, Luo J, Tang M, Zhou L, Weng Y, He TC and Zhang Y: BMP9 inhibits the proliferation and invasiveness of breast cancer cells MDA-MB-231. J Cancer Res Clin Oncol 137: 1687-1696, 2011.

22. Langenfeld E, Hong CC, Lanke G and Langenfeld J: Bone morphogenetic protein type I receptor antagonists decrease growth and induce cell death of lung cancer cell lines. PLoS One 8: e61256, 2013

23. Na YR, Seok SH, Kim DJ, Han JH, Kim TH, Jung H, Lee BH and Park JH: Bone morphogenetic protein 7 induces mesenchymal-to-epithelial transition in melanoma cells, leading to inhibition of metastasis. Cancer Sci 100: 2218-2225, 2009.

24. Leask A, Denton CP and Abraham DJ: Insights into the molecular mechanism of chronic fibrosis: the role of connective tissue growth factor in scleroderma. J Invest Dermatol 122: 1-6, 2004.

25. Cozzolino M, Biondi ML, Banfi E, Riser BL, Mehmeti F, Cusi D and Gallieni M: CCN2 (CTGF) gene polymorphism is a novel prognostic risk factor for cardiovascular outcomes in hemodialysis patients. Blood Purif 30: 272-276, 2010.

26. Kim GJ, Rhee H, Yoo JE, Ko JE, Lee JS, Kim H, Choi JS and Park YN: Increased expression of CCN2, epithelial membrane antigen, and fibroblast activation protein in hepatocellular carcinoma with fibrous stroma showing aggressive behavior. PLoS One 9: e105094, 2014.

27. Lacle MM, van Diest PJ, Goldschmeding R, van der Wall E and Nguyen TQ: Expression of connective tissue growth factor in male breast cancer: clinicopathologic correlations and prognostic value. PLoS One 10: e0118957, 2015.

28. Jacobson A and Cunningham JL: Connective tissue growth factor in tumor pathogenesis. Fibrogenesis Tissue Repair 5 (Suppl 1): S8, 2012.

29. Wazir U, Jiang WG, Sharma AK, Newbold RF and Mokbel K: The mRNA expression of inhibitors of DNA binding-1 and -2 is associated with advanced tumour stage and adverse clinical outcome in human breast cancer. Anticancer Res 33: 2179-2183, 2013.

30. Clementi C, Tripurani SK, Large MJ, Edson MA, Creighton CJ, Hawkins SM, Kovanci E, Kaartinen V, Lydon JP, Pangas SA, et al: Activin-like kinase 2 functions in peri-implantation uterine signaling in mice and humans. PLoS Genet 9: e1003863, 2013. 